\title{
Genetics Corner: An Infant with Amyoplasia Limited to the Upper Extremities
}

Ciara Catherine Fulgar, OMS III, Jared Huggins, OMS III, Aaron Ly, OMS III, Alma Zamora, OMS III, and Robin D. Clark, MD

\section{Clinical case presentation}

A pre-term male infant with congenital contractures confined to the upper extremities was referred for a genetic consultation. Due to quarantine restrictions imposed by the coronavirus pandemic, the geneticist conducted this consultation at a distance and examined photographs of the child provided by the care team. The medical students documented the history and physical examination below on site.

This infant was born at 33 weeks 3 days gestation by C-section to a 23-year old G2P1 mother following persistent non-reactive NST with decelerations. Prenatal care began at 11 weeks gestation and included a cerclage placement for a history of a previous stillbirth at 20 weeks gestation. The mother admitted to using cannabis but denied alcohol, tobacco, or any other drug use during pregnancy. Asymmetric IUGR and severe oligohydramnios complicated the prenatal course. However, fetal ultrasound examination four days before delivery did not reveal any upper or lower extremity defects. NIPT and CF screen were negative. Birth weight was 1340 grams ( $<3$ percentile), and the birth length was $41.5 \mathrm{~cm}$ (11-25 percentile). Apgar scores were 5 and 8 at 1 and 5 minutes, respectively. The newborn required resuscitation in the delivery room and was transferred to the NICU on nasal cannula CPAP, given his ongoing respiratory distress.

Physical exam revealed joint contractures at the elbows bilaterally, with an inability to flex the elbow joints. Shoulders appeared narrow, sloping, and internally rotated. There was decreased range of motion at the wrists (Figure 1). The upper extremity musculature was underdeveloped. Hips and lower extremities had a full range of motion and normal morphology. No other anomalies were noted. On neurological examination, the newborn responded to tactile stimulation, though tone and activity were decreased. The skin was pink and adequately perfused. No rashes, vesicles, or other lesions were noted. Radiographically, the right elbow was unremarkable for any pathology. Cranial ultrasound examination was normal with no evidence of significant hemorrhage.

In the NICU, occupational therapy treated the newborn for 15 minutes daily. Place-and-hold passive range of motion was performed to encourage elbow flexion and wrist rotation. Pre-treatment elbow range of motion measured at 0 degrees, bilaterally. Post-treatment, right elbow and left elbow passive range of motion were 0-15 degrees and 0-25 degrees, respectively. A flexion position was maintained at the end of the session with swaddle wrap. The baby was discharged home, tolerating feeds, and was medically stable with a recommendation for parents to continue home exercises.

The family history was noncontributory. The patient has no siblings. The parents reported Hispanic (mother) and Caucasian (father) ancestry. They denied consanguinity. There was no family history of amyoplasia or any other musculoskeletal abnormalities. There was no family history of birth defects, developmental delay, intellectual disability, or multiple miscarriages.

\section{Discussion}

This infant has amyoplasia based on the pattern of his congenital contractures - specifically the combination of sloping shoulders, upper arm extension, decreased muscle mass with replacement by fibrous tissue and fat, and limited spontaneous movement of the affected extremities. Of these, the most telling sign is the socalled "policeman's tip "position of the upper limbs in which the arms are internally rotated at the shoulders, the elbows are extended, and the wrists are flexed and usually facing the back (Figure 1). Amyoplasia is the most common form of arthrogryposis multiplex congenita, representing about $50 \%$ of cases. A clinical diagnosis should be made with care because of the low recurrence risk associated with this sporadic and non-genetic condition. The etiology of amyoplasia is not known, but it is thought to be vascular disruption in utero, leading to hypoperfusion of the spinal cord roots. Usually, all four limbs are affected. However, as Hall (2014) described in a series of 560 patients with amyoplasia, $16.8 \%$ had involvement of the upper limbs only, and $15.2 \%$ had involvement of the lower limbs only. Three limb involvement was documented in $4.8 \%$. When the lower extremities were affected, unlike the upper extremities, which have a characteristic and recognizable position, the legs were fixed in various positions, but talipes equinovarus was almost always present.

\section{"Patients with amyoplasia may have associated anomalies that share a vascular etiology, such as bowel atresias, gastroschisis, and small or partially absent digits with constriction rings and distal amputations as part of amniotic band sequence (Hall et al., 2014)."}

Patients with amyoplasia may have associated anomalies that share a vascular etiology, such as bowel atresias, gastroschisis, and small or partially absent digits with constriction rings and distal amputations as part of amniotic band sequence (Hall et al., 2014). Deep dimples in the extremities, abdominal hernias, and facial hemangiomas are reported, Bone density is reduced due to decreased fetal movement, and perinatal long bone fractures

\section{Readers can also follow} EONATOLOGY TO via our Twitter Feed @NEOTODAY 


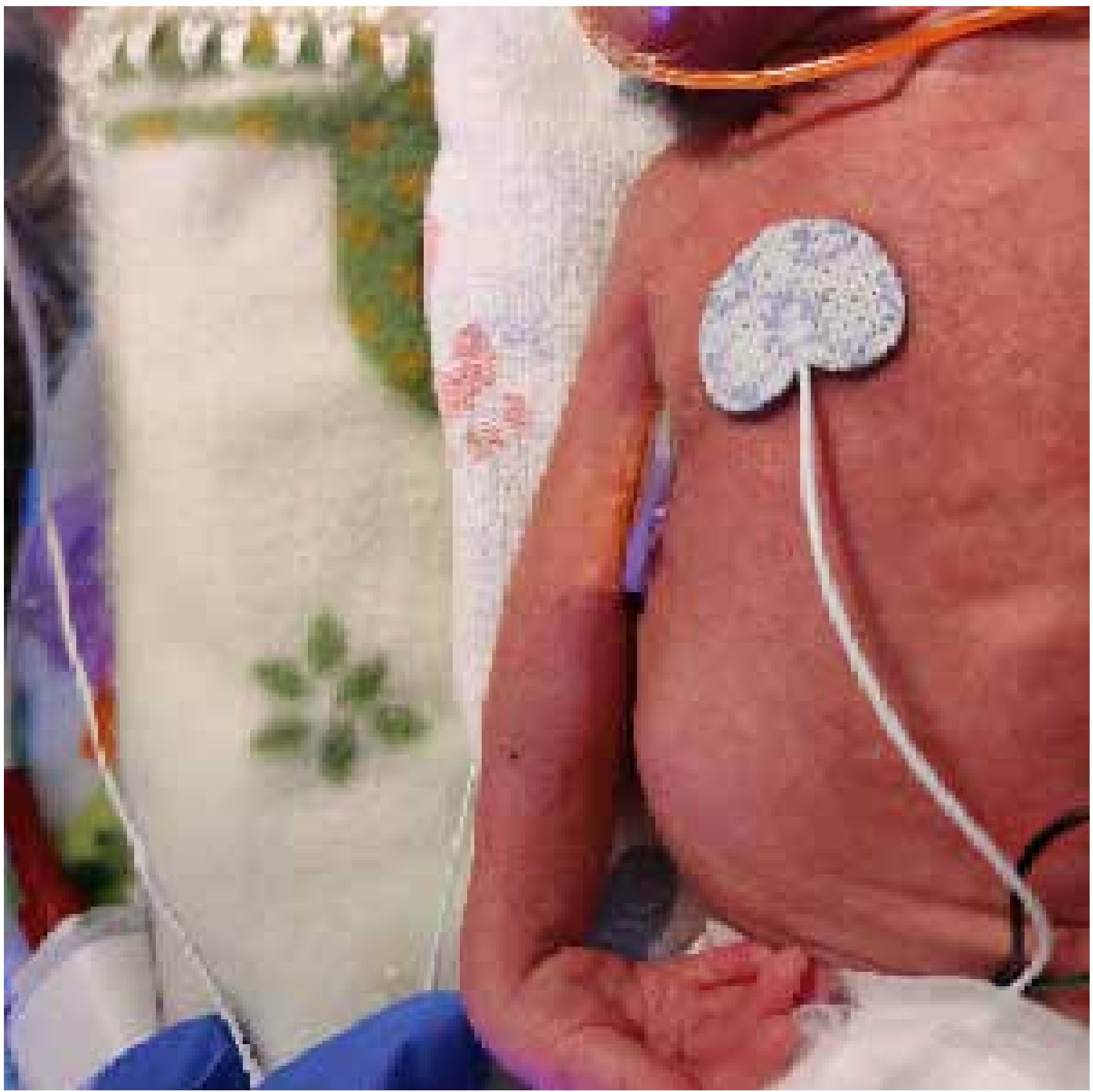

Figure 1: Note the lack of muscle mass in the upper extremity that is internally rotated at the shoulder, extended at the elbow, and flexed at the wrist. This characteristic position, known as "the policeman's tip," is virtually pathognomonic for amyoplasia.

The only worldwide monthly publication
exclusively serving Pediatric and Adult
Cardiologists that focus on Congenital/
$\begin{aligned} & \text { CONGENITAL } \\ & \text { CARDIOLOGY } \\ & \text { TODAY }\end{aligned}$$\quad \begin{aligned} & \text { Cardiothoracic Surgeons. } \\ & \text { Free on the Home Page }\end{aligned}$


occurred in $10.5 \%$ in Hall's series. Even in the absence of an amniotic band sequence, circumferential marks may be present that correspond to an umbilical cord that was tightly wrapped, in utero, around a limb with decreased mobility. An excess of discordantly affected monozygotic twins has been reported in amyoplasia. Intelligence is usually normal.

Children with amyoplasia respond well to multidisciplinary support, including physical and occupational therapy and assistive devices. The Wilmington Robotic Exoskeleton (WREX) is an articulated arm orthosis that enhances upper extremity function and improves life quality in children with neuromuscular diseases that cause weakness and limited range of motion. It negates gravity's effects with rubber bands and 3-D printable components that are made to measure, enabling the device to grow with the child. Online videos demonstrate the benefits of this technology: https:// www.youtube.com/watch?app=desktop\&v=w-4EJ1rlvv0, which is effective in children with arthrogryposis and spinal muscular atrophy (Haumont et al. 2011). I recommend sharing these videos with parents before discharge from the NICU, so parents are aware of this device for their child.

\section{Practical Applications:}

1. Be aware of the variability in amyoplasia, which can involve only the upper limbs.

2. Recognize that "the policeman's tip" position of the upper extremity in a child with arthrogryposis is a reliable sign of amyoplasia

3. Examine the infant with amyoplasia for other vascularly-mediated congenital anomalies, including bowel atresia, gastroschisis, circumferential constrictions caused by amniotic bands, and impressions on the limbs left by umbilical cord wrapping.

4. Inform the families about the availability of orthoses that improve upper extremity function and offer to show them videos demonstrating the WREX orthosis.

\section{References:}

1. Hall JG. Amyoplasia involving only the upper limbs or only involving the lower limbs with review of the relevant differential diagnoses. Am J Med Genet A. 2014 Apr;164A(4):859-73. PMID: 24459095

2. Hall JG, Aldinger KA, Tanaka KI. Amyoplasia revisited. Am J Med Genet A. 2014 Mar;164A(3):700-30. PMID: 24459070

3. Haumont T, Rahman T, Sample $W$, et al. Wilmington robotic exoskeleton: a novel device to maintain arm improvement in muscular disease. J Pediatr Orthop. 2011 JulAug;31(5):e44-9. PMID: 21654447

Disclosures: The authors have no relevant disclosures.
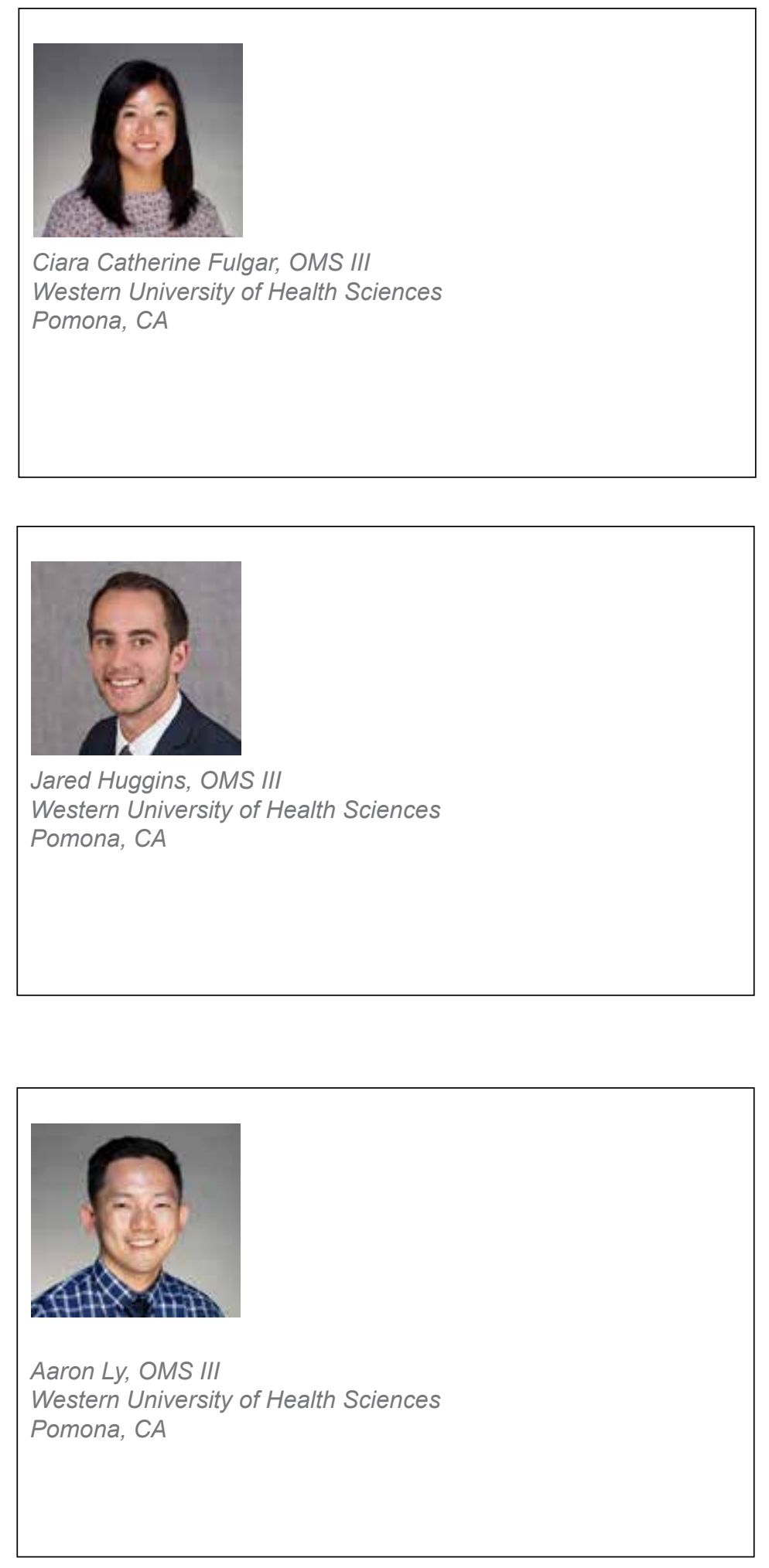

NEONATOLOGY TODAY is interested in publishing manuscripts from Neonatologists, Fellows, NNPs and those involved in caring for neonates on case studies, research results, hospital news, meeting announcements, and other pertinent topics.

Please submit your manuscript to: LomaLindaPublishingCompany@gmail.com 


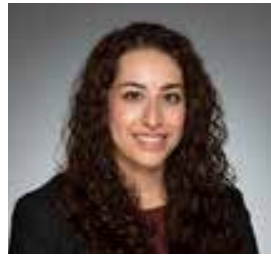

Alma Zamora, OMS III,

Western University of Health Sciences

Pomona, CA

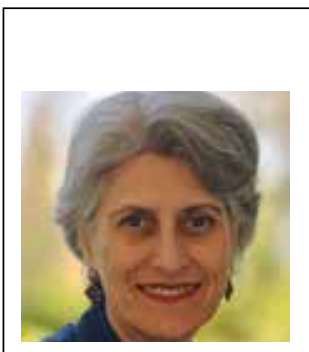

Corresponding Author

Robin Clark, MD

Professor, Pediatrics

Loma Linda University School of Medicine

Division of Genetics

Department of Pediatrics

Email rclark@llu.edu

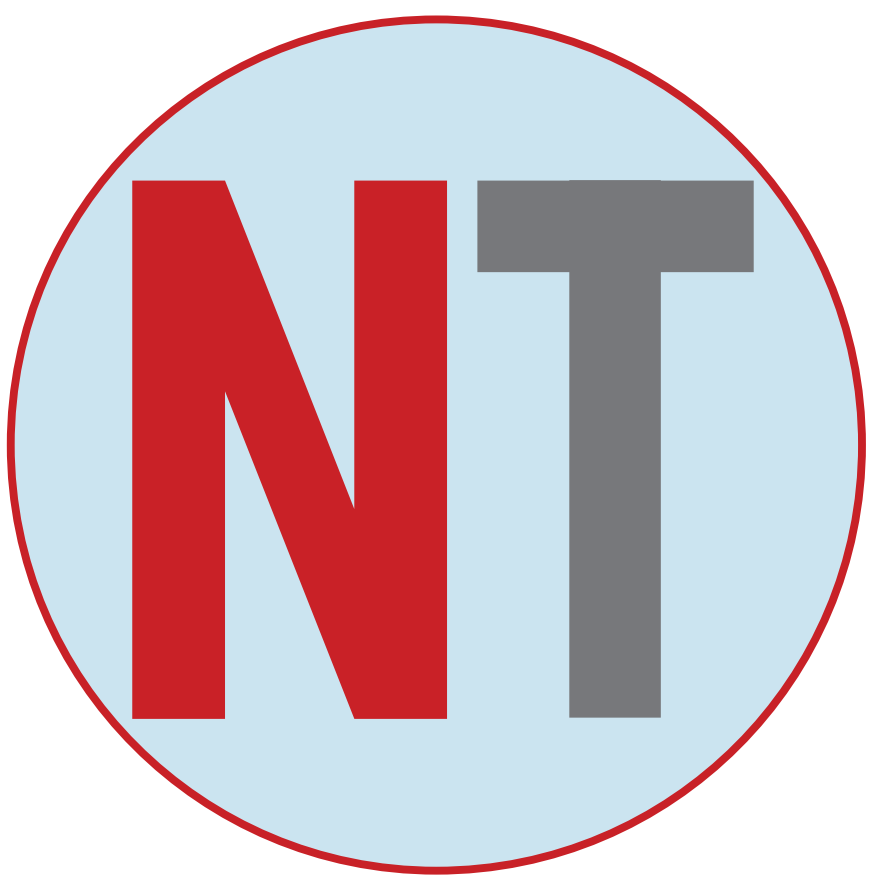

\section{OPIOIDS and NAS}

When reporting on mothers, babies, and substance use

\section{LANGUAGE MATTERS}

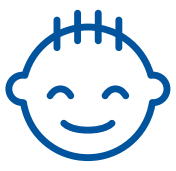

I am not an addict.

I was exposed to substances in utero. I am not addicted. Addiction is a set of behaviors associated with having a Substance Use Disorder (SUD).

\section{I was exposed to opioids.}

While I was in the womb my mother and I shared a blood supply. I was exposed to the medications and substances she used. I may have become physiologically dependent on some of those substances.

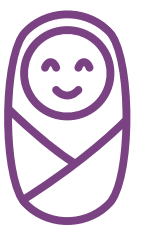

\section{NAS is a temporary and} treatable condition.

There are evidence-based pharmacological and non-pharmacological treatments for Neonatal Abstinence Syndrome.

\section{My mother may have a SUD.}

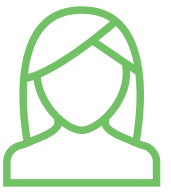

She might be receiving Medication-Assisted Treatment (MAT). My NAS may be a side effect of her appropriate medical care. It is not evidence of abuse or mistreatment.

\section{My potential is limitless.}

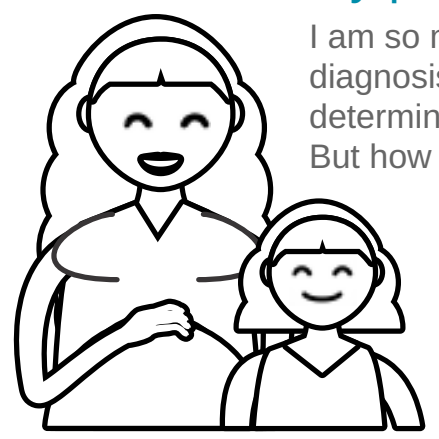
am so much more than my NAS diagnosis. My drug exposure will not determine my long-term outcomes. But how you treat me will. When you invest in my family's health and wellbeing by supporting Medicaid and Early Childhood Education you can expect that I will do as well as any of my peers!

Learn more about

Neonatal Abstinence Syndrome at WWW.nationalperinatal.org 